\title{
A utilização do laser em Ortodontia
}

\author{
Leniana Santos Neves*, Cíntia Maria de Souza e Silva**, José Fernando Castanha Henriques***,

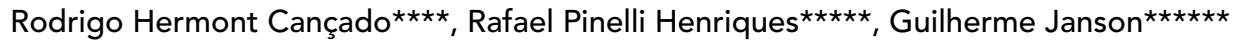

\begin{abstract}
Resumo
O laser vem sendo amplamente utilizado na área da saúde, encontrando-se em franca evolução na Odontologia, beneficiando o paciente com tratamentos atraumáticos, sem dor, com melhor pós-operatório, entre muitas outras vantagens. A Ortodontia também tem muito a ganhar com a utilização da luz laser, apesar de suas aplicações e seus efeitos não se encontrarem bem difundidos entre os profissionais. $O$ presente trabalho objetivou realizar uma revisão na literatura a fim de elucidar o ortodontista sobre como ele poderá se beneficiar desta forma de energia, elevando a qualidade do seu trabalho e melhorando as condições do tratamento tanto para o profissional como para o paciente.
\end{abstract}

Palavras-chave: Lasers. Ortodontia. Odontologia.

\section{INTRODUÇÃO}

A palavra laser é uma abreviatura para "Light Amplification of Stimulated Emission of Radiation" que na Língua Portuguesa significa Amplificação da Luz por Emissão Estimulada de Radiação. O laser é uma radiação que se encontra no espectro de luz que varia do infravermelho ao ultravioleta,

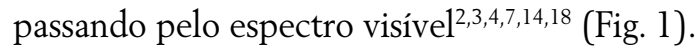

A utilização terapêutica da energia luminosa vem desde os primórdios da civilização, e em 1903, o prêmio Nobel de medicina foi destinado ao Dr. Nielo Ryberg Finsen pelo tratamento realizado com a luz solar em um paciente que apresentava um tipo de tuberculose de pele ${ }^{3}$. Albert Einstein, em 1916, formulou os princípios da amplificação da luz por emissão estimulada de radiação, quando percebeu em seu experimento que a emissão induzida poderia existir e a radiação eletromagnética seria produzida por um processo atômico ${ }^{2,3,4,7,14}$. No ano de 1960, Theodoro H. Maiman desenvolveu o primeiro aparelho emissor de laser, a cristal de rubi, que passou a ser comercializado. No ano seguinte foi realizada a primeira intervenção cirúrgica com o laser, no Hospital Presbiteriano de Nova York, para a retirada de um pequeno tumor de retina que impedia a visão. Em 1965, Sinclair e Knoll desenvolveram o laser terapêutico, não mais com efeito de corte, mas de bioestimulação dos tecidos ${ }^{2,3,4}$.

* Mestre em Ortodontia pela Faculdade de Odontologia de Bauru - USP; Aluna do curso de Pós-graduação em Ortodontia, nível Doutorado da Faculdade de Odontologia de Bauru - USP.

** Cirurgiã-Dentista graduada pela Universidade do Sagrado Coração - Bauru - SP. Ex-aluna do Curso de Aperfeiçoamento em Ortodontia da ACOPEN (Assessoria e Consultoria em Ortodontia, Pesquisa e Ensino) - Bauru - SP. Auxiliar do Curso de Aperfeiçoamento em Ortodontia da ACOPEN - Bauru - SP.

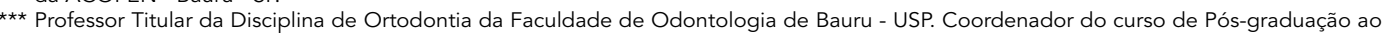
nível de Doutorado em Ortodontia pela Faculdade de Odontologia de Bauru - USP. Prefeito do campus da USP de Bauru.

$\star \star \star \star$ Mestre em Ortodontia pela Faculdade de Odontologia de Bauru. USP. Aluno do curso de Pós-graduação em Ortodontia, nível Doutorado da Faculdade de Odontologia de Bauru - USP.

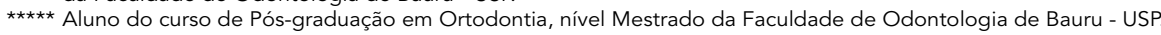

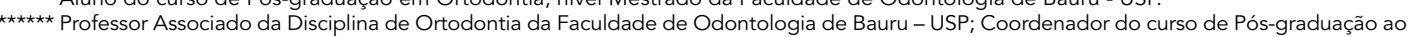
nível de Mestrado em Ortodontia pela Faculdade de Odontologia de Bauru - USP; Membro do "Royal College of Dentists of Canada". 


\section{CARACTERÍSTICAS DA LUZ LASER}

A luz laser oferece uma segurança relevante ao ser utilizada, e difere das outras formas de luz devido principalmente a três características:

- A monocromaticidade (a luz laser é composta de fótons, todos da mesma cor e com o mesmo comprimento de onda. É, portanto, uma luz pura. Essa característica é importante devido à absorção seletiva do tecido humano) ${ }^{4,7,14}$ (Fig. 2).

- A coerência (as ondas viajam ordenadamente em relação ao tempo e suas amplitudes são iguais. A coerência mantém-se ao longo do tempo e espaço) $)^{4,7,14}$ (Fig. 3).

- A unidirecionalidade ou colimação (o feixe de fótons é paralelo ao eixo do tubo que produz este tipo de energia; a luz laser possui divergência angular muito pequena, toda a energia do laser concentra-se precisamente em um ponto focal) ${ }^{4,7,14}$ (Fig. 4).

\section{CLASSIFICAÇÃO DOS LASERS E MODO DE AÇÃO}

Os aparelhos de laser são constituídos por um meio ativo, que pode ser sólido (Rubi), gasoso (mais comuns, como exemplo o $\mathrm{CO}_{2}$, $\mathrm{He}-\mathrm{Ne}, \mathrm{Ar}$ ), semicondutor (Diodo - AsGaAl, AsGa), semisólido (Nd-YAG, Er-YAG, YAP), Excímero ( $\mathrm{KrF}$, $\mathrm{XeCl}$ ) ou líquido (pouco usado, como exemplo, rodamine e cumarina - Dy laser) $)^{3,4,7,14}$.

Os lasers são classificados de acordo com a potência de emissão da radiação podendo ser: laser de alta, média e baixa intensidade.

Os lasers de alta intensidade, também conhecidos como laser cirúrgico, laser quente, laser duro ou hard laser emitem radiação de alta potência, o que propicia um potencial destrutivo, sendo utilizados para viabilizar cirurgias ou remoção de tecido cariado, ou seja, possui uma ação fototérmica de corte, vaporização, coagulação e esterilização dos tecidos. Os principais lasers de alta intensidade são o Excimer, Argônio, Kripton, Dye, Rubi, Família YAG (itrio-alumínio-granada) e $\mathrm{CO}_{2}^{3,4,7,14}$.

Os lasers de média intensidade ou mid-laser

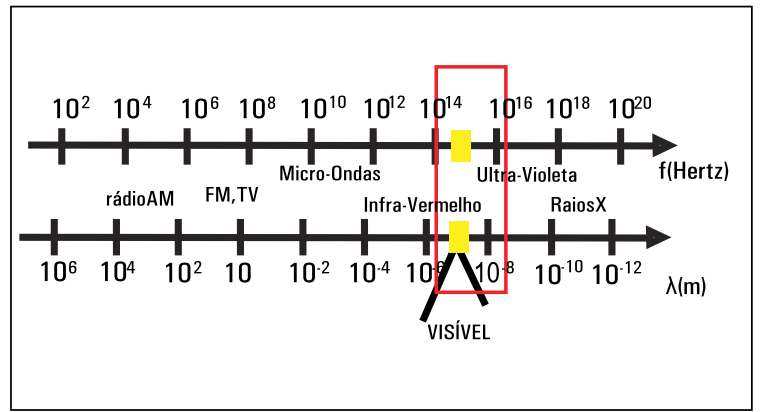

FIGURA 1 - Espectro eletromagnético, evidenciando onde se encontra a faixa relacionada ao laser.

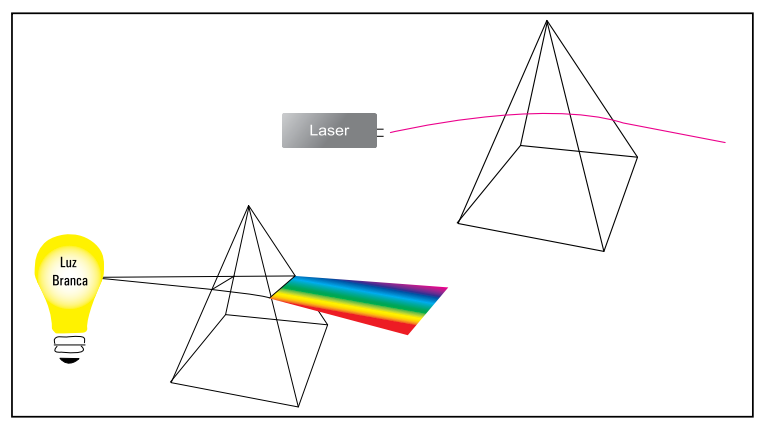

FIGURA 2 - Característica da luz laser de monocromaticidade.

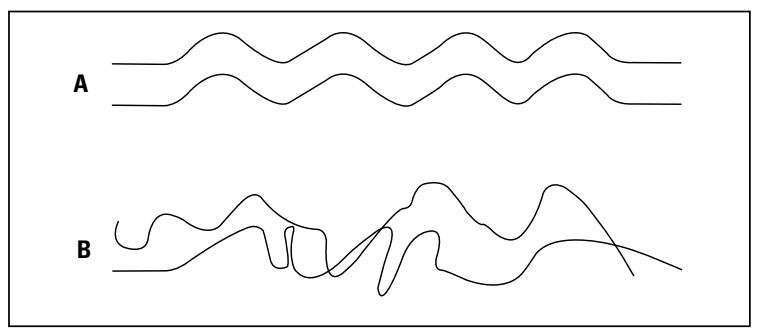

FIGURA 3 - Característica de coerência da luz laser. Em $\mathbf{A}$, comprimentos de ondas coerentes no tempo e espaço, em $\mathbf{B}$, comprimentos de ondas incoerentes no tempo e espaço.

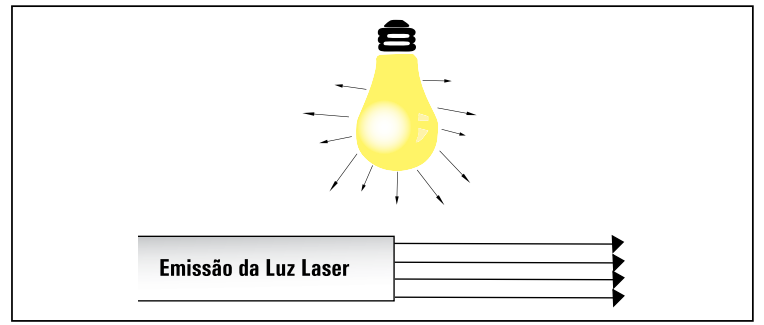

FIGURA 4 - Característica da luz laser de unidirecionalidade ou colimação. A luz natural é divergente e a luz laser é paralela ao tubo onde é produzida. 
emitem radiações com potências medianas, sem poder destrutivo, sendo mais utilizados em fisioterapia. Entre eles se encontram o laser de HélioNeônio (He-Ne) e o Arseniato de gálio (AsGa) ${ }^{7}$.

Os lasers de baixa intensidade, também denominados laser mole, laser frio, laser terapêutico ou "soft-laser", emitem radiações de baixas potências, sem potencial destrutivo, e possuem uma ação fotoquímica de analgesia, anti-inflamatória e de bioestimulação tecidual. Entre os lasers de baixa intensidade encontra-se os lasers: He-Ne (HélioNeônio), diodo (Arseniato de gálio - AsGa e Arseniato de gálio e alumínio - AsGaAl) 3,4,7,14.

\section{DOSIMETRIA}

A dosimetria consiste na relação entre a energia transmitida por um emissor laser e a superfície de irradiação do raio de luz, podendo ser expressa em joules por centímetro quadrado $\left(\mathrm{J} / \mathrm{cm}^{2}\right)$. Esta expressão é utilizada quando se fala em dose de tratamento. O tempo de exposição da maioria dos aparelhos atuais possui um cálculo direto, onde o profissional programa no equipamento a DE (densidade energética) e o aparelho mostra o tempo de exposição em função da potência de emissão e da área irradiada ${ }^{2,3,7,14}$. Existem alguns parâmetros de valores de densidade energética em função do efeito desejado como $^{7,14}$ :

- efeito antiálgico - de 2 a $4 \mathrm{~J} / \mathrm{cm}^{2}$

- efeito anti-inflamatório de 1 a $3 \mathrm{~J} / \mathrm{cm}^{2}$

- efeito regenerativo de 3 a $6 \mathrm{~J} / \mathrm{cm}^{2}$

- efeito circulatório de 1 a $3 \mathrm{~J} / \mathrm{cm}^{2}$

Quando desejamos um efeito anti-inflamatório, o calculo da DE deve basear-se na característica da inflamação, ou seja, para uma inflamação aguda as doses devem ser baixas, em torno de 1 a 3 $\mathrm{J} / \mathrm{cm}^{2}$, uma inflação subaguda deve-se utilizar doses médias de 3 a $4 \mathrm{~J} / \mathrm{cm}^{2}$, já na inflamação crônica necessita-se de doses altas, de 5 a $7 \mathrm{~J} / \mathrm{cm}^{2}$. Porém, esses parâmetros devem ser determinados pelo profissional, que pode variar ou alterar as doses de aplicação em função da resposta do paciente e/ou do tipo de lesão $0^{2,7,14}$.

\section{CUIDADOS A SEREM OBSERVADOS QUAN- DO DA UTILIZAÇÃO DO LASER}

A partir dos anos 80 foram estabelecidas algumas normas de segurança para a utilização dos aparelhos a laser ${ }^{14}$. De acordo com as normas éticas, o profissional não deve garantir resultados favoráveis aos pacientes; deve-se ter cuidado com os efeitos secundários, principalmente nos olhos e pele ${ }^{3,4,14}$. $\mathrm{O}$ raio laser pode provocar alterações sistêmicas indesejáveis, principalmente quando aplicado em glândulas, como por exemplo, a tireóide ${ }^{14}$.

Em relação às normas de segurança, os aparelhos de laser são classificados quanto ao espectro eletromagnético e seus riscos em provocar lesões oculares: luz visível (pode provocar lesões na retina), infravermelho (pode provocar lesões na córnea e cristalino), ultravioleta (pode provocar alterações celulares - citoplasma e núcleo - podendo provocar queimaduras ou câncer) ${ }^{14}$. Quando o feixe de luz laser é focalizado no olho, podese provocar queimaduras na retina com lesões irreversíveis de alteração visual. Portanto, torna-se indispensável a utilização de óculos de proteção pelo profissional, auxiliar e paciente (Fig. 5), sendo que para cada comprimento de onda existe um tipo de óculos de proteção específico ${ }^{2,3,4,7,14}$.

Um outro cuidado que se deve tomar quando se utiliza o laser é nunca permitir a entrada de qualquer pessoa no ambiente com o aparelho ligado e em funcionamento, pois o operador pode desviar sua atenção e provocar um acidente; assim, as portas de acesso aos ambientes com laser devem constar sempre avisos sobre o comprimento de onda que está sendo utilizado e a sala deve conter uma chave geral para bloqueio de corrente elétrica caso a porta seja aberta inadvertidamente ${ }^{3,14}$.

$\mathrm{Na}$ sala deve-se evitar a presença de qualquer tipo de material refletor, como instrumentais (deve-se utilizar instrumentais plásticos, e não metálicos), anéis, relógios e outros ${ }^{3,4}$.

Deve-se questionar ao paciente se ele faz uso de substâncias químicas fotossensiveis, como o ácido retinóico, tetraciclina, gliseofulvinas, sulfaminas e 

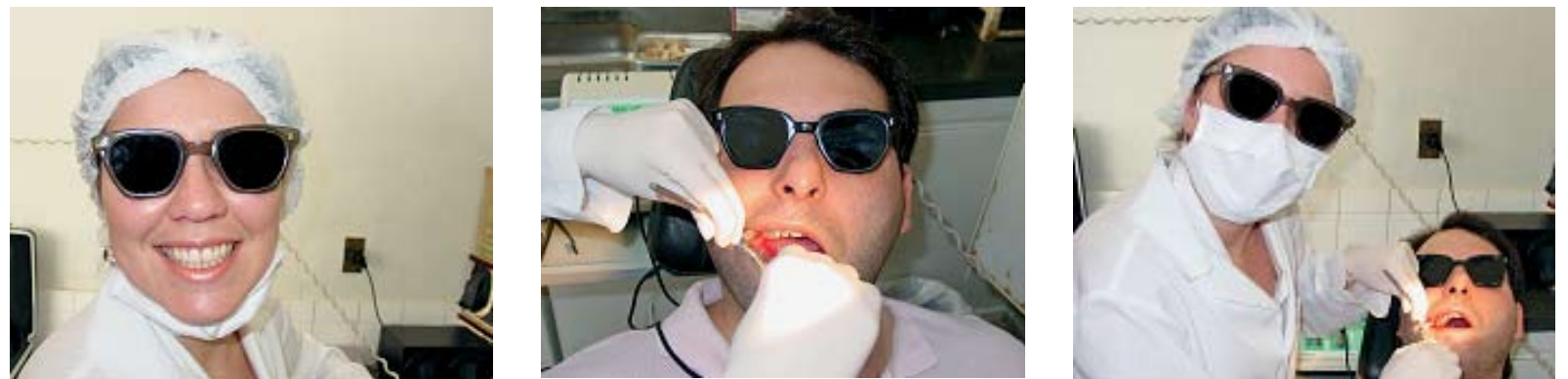

FIGURA 5 - Utilização de óculos de proteção pelo profissional e paciente. A auxiliar também deve utilizar os óculos de proteção. Para cada comprimento de onda existe um tipo de óculos.

as furocumarinas, que poderão induzir reações de fotossensibilização na pele, manifestadas por meio de manchas cutâneas nas áreas irradiadas, que tendem a desaparecer após a interrupção do tratamento ${ }^{3,4,14}$.

Existem algumas restrições para os lasers de baixa potência, como nos casos de portadores de marca-passo, epiléticos, em irradiações diretas em glândulas endócrinas e globo ocular, e em pacientes com neoplasias ${ }^{2,14}$.

\section{ORIENTAÇÕES PRELIMINARES À APLICA- ÇÃO DO LASER}

Em aplicações extrabucais, deve-se preocupar com a limpeza da pele do paciente, removendo resíduos de maquiagem e cremes que se comportam como barreiras físicas para a absorção da luz laser. Deve-se medir a extensão da lesão com régua para o correto cálculo da dosimetria. Em aplicação intrabucal, a superfície a ser irradiada deverá estar necessariamente limpa e seca, sendo sempre necessário utilizar o isolamento relativo. $\mathrm{O}$ ângulo de incidência do raio sobre o tecido deverá ser o mais perpendicular possível para minimizar a refração do raio no tecido. As áreas metálicas (restaurações, instrumental metálico, etc.) não devem ser irradiadas para evitar a reflexão do raio, que impedirá a absorção da luz laser. Recomenda-se no início do tratamento utilizar a menor dose terapêutica total por sessão, aumentando gradativamente quando necessário. As respostas ao tratamento geralmente são observadas em média após a terceira sessão te- rapêutica, exceto no tratamento das lesões neurossensoriais, neuromotoras e regeneração óssea. Recobrir a ponteira do laser com uma única camada de filme de PVC para proteger o aparelho dos fluidos salivares e também o paciente da contaminação. A laserterapia pode ser um tratamento isolado ou coadjuvante a um tratamento convencional ${ }^{2,3}$.

\section{APLICAÇÃO DO LASER NA ORTODONTIA}

A utilização da luz laser vem se tornando cada vez mais freqüente na clínica odontológica, trazendo benefícios inquestionáveis ao pacientes nas diversas especialidades odontológicas. A Ortodontia também pode se beneficiar dos efeitos da laserterapia, apesar disto ainda não estar bem difundido entre os profissionais desta área. Pode-se utilizar o laser em Ortodontia com as finalidades de: descolagem de braquetes cerâmicos; reparação óssea após a expansão rápida da maxila; odontalgia decorrente da movimentação ortodôntica; polimerização da resina durante a colagem de braquetes; holografia, que permite o armazenamento das imagens dos modelos de gesso; scanner a laser permitindo a utilização de imagens tridimensionais no diagnóstico e planificação do tratamento ortodôntico ou ortodôntico/cirúrgico; diagnóstico de lesão de mancha branca em pacientes ortodônticos; inter-relação Ortodontia e Periodontia e reparo das úlceras traumáticas originadas pelos acessórios ortodônticos. A seguir, será detalhada cada uma destas aplicações da radiação laser em Ortodontia. 

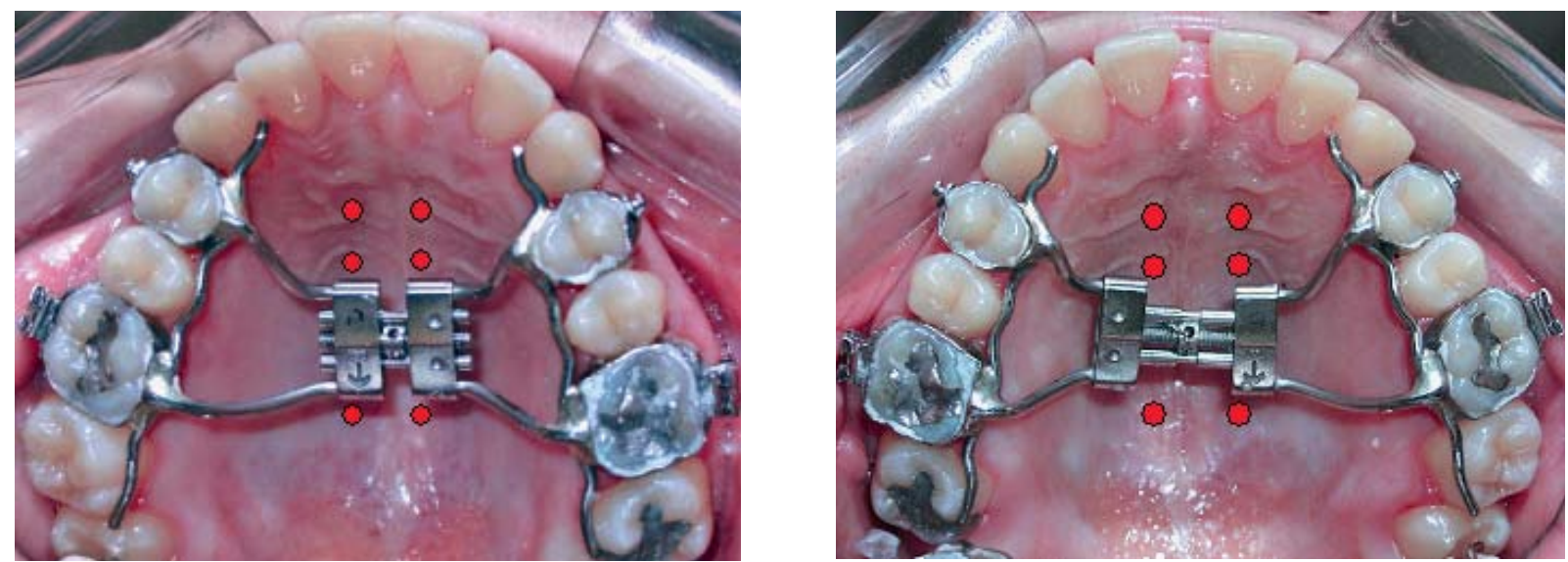

FIGURA 6 - Pontos de aplicação do laser ao longo da sutura palatina mediana nos casos de expansão rápida da maxila.

\section{Descolagem de braquetes cerâmicos}

Na descolagem de braquetes cerâmicos, o laser de alta potência produz calor, que causa um amolecimento da resina e facilita a remoção do braquete sem que ocorra a fratura do esmalte dentário, nem danos pulpares ${ }^{5,8,13,15,17,21}$. Para esse procedimento, utiliza-se o laser de alta intensidade do grupo YAG ou, na maioria das vezes, o laser de $\mathrm{CO}_{2}$ (com comprimento de onda de $10,6 \mu \mathrm{m}$, numa potência de 3 a $20 \mathrm{~W}$ com tempo de 2 a 3 segundos, de forma pulsátil) ${ }^{13,15}$.

\section{Expansão rápida da maxila}

A ação do laser após a realização da expansão rápida da maxila consiste na diminuição da dor ou desconforto do paciente que pode ser decorrente deste procedimento, além de acelerar a reparação óssea ${ }^{3,20}$. Saito e Shimizu ${ }^{20}$ mostraram que o osso neoformado apresenta uma qualidade superior ao osso formado quando não se utiliza o raio laser. Como a reparação óssea torna-se acelerada com a laserterapia, conseqüentemente o tempo de contenção pode ser diminuído. Para essa finalidade utiliza-se o laser de baixa intensidade Diodo de Arseniato de gálio alumínio, com aplicações em pontos determinados distribuídos na trajetória paralela à sutura palatina mediana, antes da ativação do expansor, com uma dose de
$2 \mathrm{~J} / \mathrm{cm}^{2}$ por ponto determinado (Fig. 6). Durante o período de ativação do expansor as aplicações devem ser realizadas de 2 a 3 sessões semanais com intervalo mínimo de 48 horas entre as mesmas, conforme a sensibilidade individual do paciente. Visando a reparação óssea, deve-se continuar as aplicações duas vezes por semana pelo período de oito semanas ${ }^{3}$.

\section{Odontalgia decorrente da movimentação ortodôntica}

No tratamento da odontalgia decorrente da movimentação ortodôntica, o laser possui uma ação analgésica, anti-inflamatória e de reparação dentinária ${ }^{3,12}$. O laser utilizado nesses casos pode ser o laser Diodo Arseniato de gálio e alumínio 3,12, sendo aplicado de forma pontual, no ápice radicular com dosimetria de $2 \mathrm{~J} / \mathrm{cm}^{2}$ e ao longo do eixo da raiz com 3 pontos de $1 \mathrm{~J} / \mathrm{cm}^{2}$ (Fig. 7). A freqüência de aplicação é de 1 a 2 sessões semanais ${ }^{3}$.

\section{Polimerização da resina durante a colagem de braquetes}

O laser utilizado para a polimerização de resina é o laser de alta intensidade de Argônio que, além da polimerização da resina, aumenta a resistência do esmalte à formação de mancha bran$\mathrm{ca}^{16,22}$. O laser de Argônio possui um comprimen- 
to de onda de 454 a $514 \mathrm{~nm}$, uma potência de 200 a $300 \mathrm{~mW}$, sendo utilizado para polimerização da resina ortodôntica por um tempo de 5 segundos, o que equivale a 40 segundos da luz convencional ${ }^{9,11,16,22}$. Assim, com a utilização desta luz laser na polimerização da resina durante as colagens dos acessórios ortodônticos, além da considerável economia de tempo clínico, há uma maior resistência do esmalte ao desenvolvimento de mancha branca ao redor dos acessórios, que pode ocorrer principalmente quando o paciente não é colaborador e não apresenta uma boa higiene bucal.

\section{Holografia}

A holografia utiliza a luz laser (He-Ne) para reproduzir tridimensionalmente e com alta qualidade a imagem dos modelos de gesso, permitindo uma análise tridimensional, a sobreposição e o armazenamento dos modelos ${ }^{18,19}$. O holograma é resistente e apresenta aproximadamente o mesmo tamanho de radiografias e fotografias. A vantagem é que substitui o armazenamento dos próprios modelos de gesso, que são frágeis, pesados, e requerem grande espaço para armazenamento. Atualmente o holograma apresenta algumas desvantagens ao medir a sobressaliência, a sobremordida e medidas de profundidade, pois nesses casos as medidas não são tão precisas ${ }^{18,19}$.

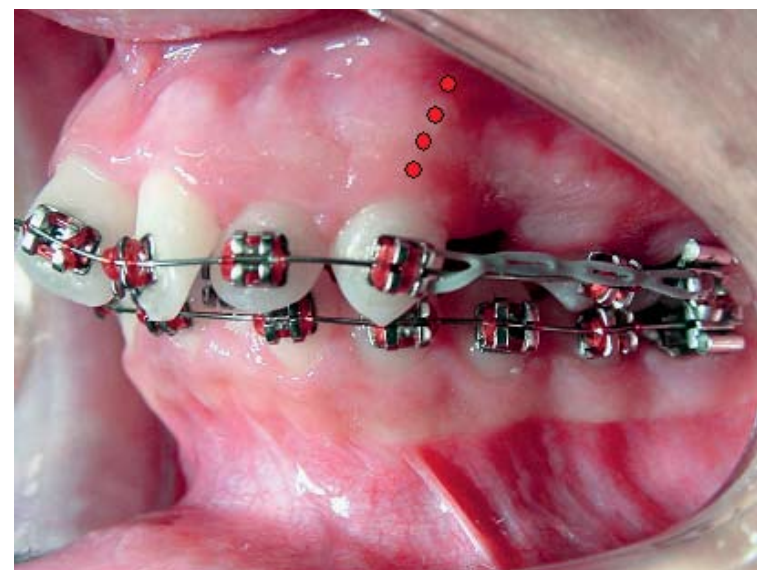

FIGURA 7 - Pontos de aplicação do laser ao longo da raiz dentária nos casos de odontalgia, após a ativação do aparelho ortodôntico fixo.

\section{Scanner a laser}

O scanner a laser utiliza o laser de baixa potência $(\mathrm{He}-\mathrm{Ne})^{10,18}$. O laser é irradiado ao longo da face ou corpo do paciente e a luz refletida é capturada por meio de uma câmera de vídeo. A imagem então pode ser vista tridimensionalmente na tela do computador e movimentada em todas as direções. Permite a sobreposição das imagens seriadas obtidas, possibilitando uma avaliação longitudinal do crescimento facial ou de uma cirurgia facial ${ }^{10,18}$.

\section{Diagnóstico de lesão de mancha branca em pacientes ortodônticos}

O diagnóstico de lesão de mancha branca é realizado com o laser de Argônio que possui comprimento de onda de $488 \mathrm{~nm}$ com potência de 10 a $20 \mathrm{~mW} / \mathrm{cm}^{2}$. Ao irradiar a luz laser, observa-se uma imagem fluorescente, com uma cor diferente (mais escura) na região da lesão de mancha bran$\mathrm{ca}^{1}$. A quantidade da fluorescência permite um adequado monitoramento das alterações minerais nas lesões de esmalte incipientes, de grande valor para a avaliação das medidas preventivas a serem utilizadas em pacientes propensos ao desenvolvimento de lesões cariosas, como é o caso de pacientes ortodônticos não colaboradores e com higienização bucal deficiente ${ }^{1}$.

\section{Ortodontia X Periodontia}

Não é raro o fato de pacientes ortodônticos apresentarem alterações periodontais, entretanto, na maioria das vezes, essas alterações são discretas e reversíveis, sendo controladas por meio de raspagens e profilaxias. Porém, em algumas situações mais graves, o paciente ortodôntico deve ser encaminhado ao periodontista para o controle e tratamento da doença periodontal apresentada. Nesses casos, o periodontista poderá intervir cirurgicamente, muitas vezes utilizando a radiação laser, que tem se mostrado bastante eficaz nesses procedimentos, realizando cirurgias praticamente sem dor e com um pósoperatório bem mais cômodo para o paciente ${ }^{6,14}$. 

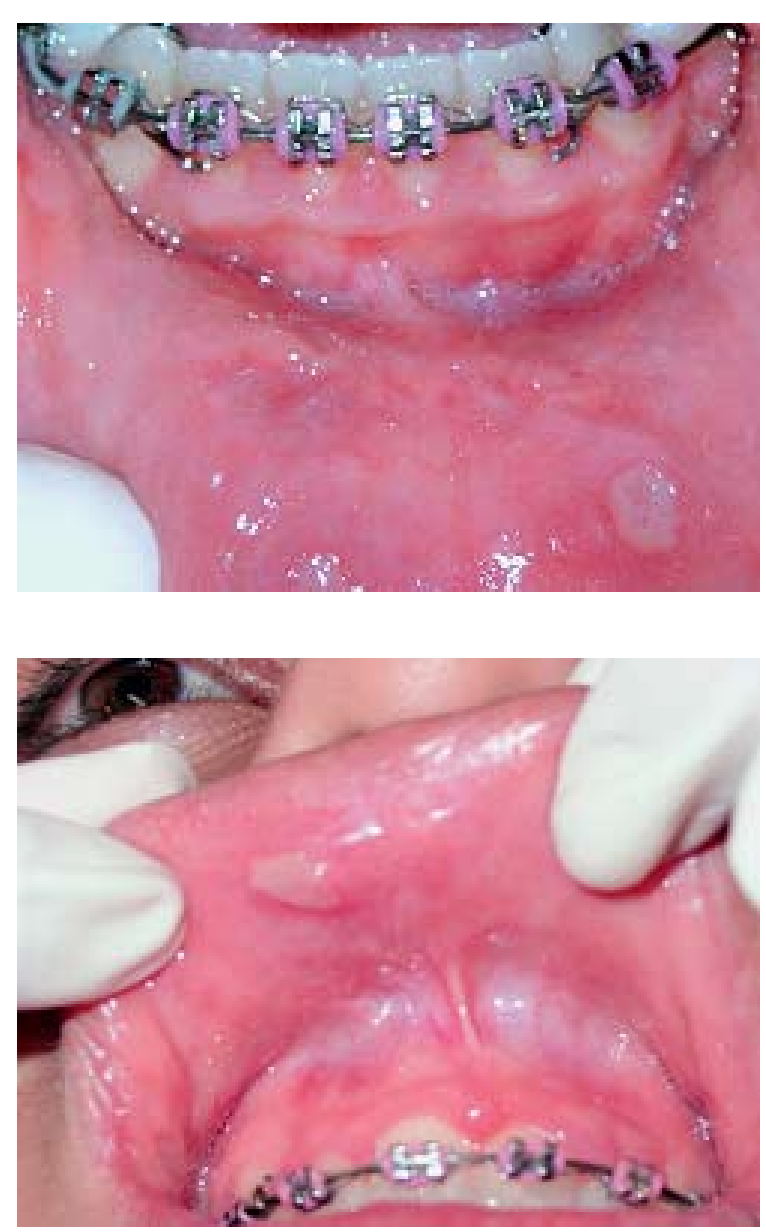

FIGURA 8 - Lesões traumáticas em pacientes com aparelhos fixos.

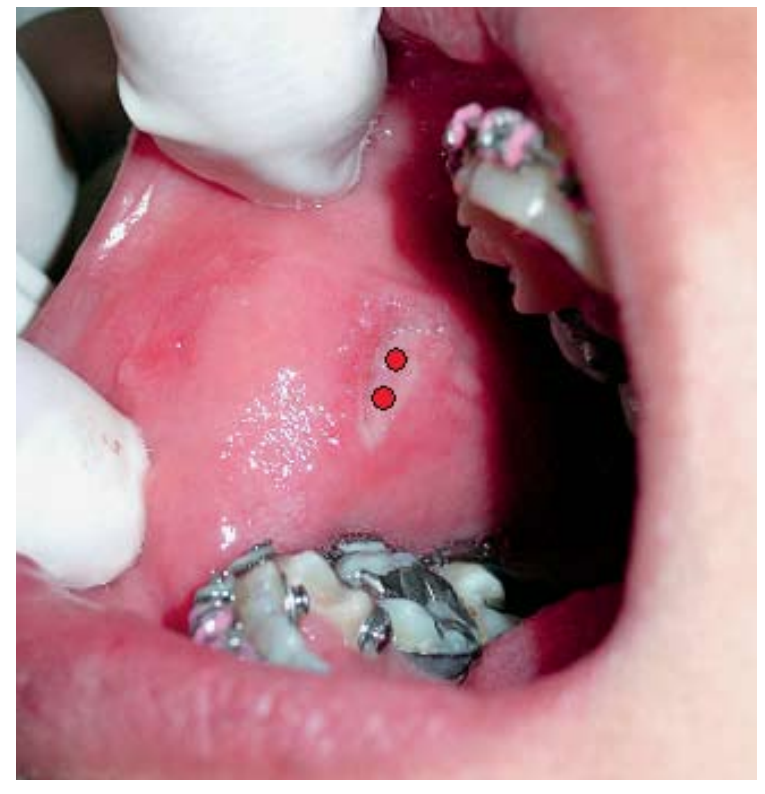

FIGURA 9 - Pontos de aplicação do laser ao longo da lesão traumática.
Para exemplificar essa situação, J.B. Mello e G.P.S. Mello ${ }^{14}$ relataram o caso de um paciente com 15 anos de idade que apresentava gengivite marginal, com comprometimento das papilas interdentais, provocada por deficiência de higienização. $\mathrm{O}$ ortodontista encaminhou a paciente para tratamento periodontal e remoção do freio labial superior. Utilizou-se o laser de Nd:YAG com $6 \mathrm{~W}$ e $50 \mathrm{~Hz}$ para cirurgia periodontal. Assim, a seleção do tipo de laser a ser utilizado bem como da dosimetria adequada, fica a critério do periodontista. Ao ortodontista cabe diagnosticar a alteração periodontal e encaminhar ao profissional qualificado.

\section{Úlceras traumáticas}

É bastante comum o surgimento de úlceras traumáticas nas mucosas dos pacientes que utilizam o aparelho ortodôntico fixo (Fig. 8). Nesses casos o laser atua com um efeito analgésico, antiinflamatório e reparador tecidual. Sua aplicação deve ser pontual no centro da lesão, para efeito analgésico, e de varredura ao longo da lesão com sobre-extensão de $0,5 \mathrm{~cm}$ para estimular a reparação, com uma dosimetria de $2 \mathrm{~J} / \mathrm{cm}^{2}$ no centro da lesão, e de 2 a $4 \mathrm{~J} / \mathrm{cm}^{2}$ ao redor da mesma ${ }^{3}$ (Fig. 9), sendo aplicados com a freqüência de 2 a 3 vezes por semana, com intervalo de 24 horas entre as sessões até a melhora da sintomatologia dolorosa e total reparação ${ }^{3}$.

\section{CONCLUSÕES}

A luz laser vem sendo cada vez mais empregada na Odontologia, mostrando-se eficaz e benéfica quando bem utilizada. Sabe-se que o laser apresenta uma grande diversidade de indicações, podendo ser utilizado com efeitos de corte, de analgesia, de estimulação tecidual, entre vários outros; basta $o$ profissional conhecer bem os tipos de lasers e o modo de ação de cada um deles. Na Ortodontia, a laserterapia também apresenta suas indicações, podendo contribuir sobremaneira com a evolução do tratamento, trazendo benefícios para o profissional e para o paciente. Porém, mais estudos devem 
ser realizados para verificar com mais detalhes as indicações e as possiveis contra-indicações desse meio auxiliar no tratamento ortodôntico.

Enviado em: Julho de 2004 Revisado e aceito: Março de 2005

\title{
Lasers in Orthodontics
}

\begin{abstract}
Laser has been widely employed in health specialties, and its application in Dentistry is currently being evolved to benefit the patient with atraumatic and painless treatments, with better post-operative recovery, besides other advantages. Orthodontics may also be benefited from the utilization of laser, even though its application and effects are not well known by professionals. The aim of the present work is to present a literature review to provide orthodontists with information on how they may apply this energy to improve the quality of their work and the conditions for treatment, both for the professional and the patient.
\end{abstract}

Key words: Lasers. Orthodontics. Dentistry

\section{REFERÊNCIAS}

1. AL-KHATEEB, S.; FORSBERG, C. M.; ANGMAR-MANSSON, B. A longitudinal laser fluorescence study of white spot lesions in orthodontic patients. Am J Orthod Dentofacial Orthop, St. Louis, v. 113, no. 6, p. 595-602, 1998.

2. BASSANI, A. C.; BASTOS, J. R. M. Considerações sobre o raio laser e sua aplicação em Odontologia. Odontologia Capixaba, Vitória, v. 20, n. 21, p. 36-39, 1992

3. BRUGNERA JÚNIOR, A.; SANTOS, A. E. C. G.; BOLOGNA, E. D.; LADALARDO, T. C. C. G. P. Atlas de laserterapia aplicada à clínica odontológica. 1. ed. São Paulo: Ed. Santos, 2003.

4. BRUGNERA JÚNIOR, A.; VILLA, R. G.; GENOVESE, W. J. Laser na Odontologia. 1. ed. São Paulo: Pancast,1991.

5. DUMORE, T.; FRIED, D. Selective ablation of orthodontic composite by using sub-microsecond IR laser pulses with optical feedback. Lasers Surg Med, New York, v. 27, no. 2, p.103-110, 2000.

6. FINKBEINER, R. L.; NELSON, L. S.; KILLEBREW, J. Accidental orthodontic elastic band-induced periodontitis: orthodontic and laser treatment. J Am Dent Assoc, Chicago, v. 128, no. 11 , p.1565-1569, 1997.

7. GENOVESE, W. J. Laser de baixa intensidade: aplicações terapêuticas em Odontologia. 1. ed. São Paulo: Lovise, 2000.

8. HIRAYAMA, J.; MARTINS, D. R.; PICOSSE, L. R.; COTRIM-FERREIRA, F. A. ; SCAVONE JÚNIOR, H.; LIBERTI, E. A. Aspecto do esmalte dental após a remoção de braquete cerâmico com o auxílio do Laser de $\mathrm{CO}_{2}$. Rev APCD, São Paulo, v. 55, n. 6, p. 440-444, 2001.

9. KURCHAK, M.; DESANTOS, B.; POWERS J.; TURNER, D. Argon laser for light-curing adhesives. J Clin Orthod, Boulder, v. 31, no. 6, p. 371-374, 1997.

10. KUSNOTO, B.; EVANS, C. A. Reliability of a 3D surface laser scanner for orthodontic applications. Am J Orthod Dentofacial Orthop, St. Louis, v. 122, no. 4, p. 342-348, 2002

11. LALANI, N.; FOLEY, T. F.; VOTH, R.; BANTING, D.; MAMANDRAS, A. Polymerization with the argon laser: curing time and shear bond strength. Angle Orthod, Appleton, v. 70, no. 1, p. $28-33,2000$

12. LIM, H. M.; LEW, K. K.; TAY, D. K. A clinical investigation of the efficacy of low level laser therapy in reducing orthodontic postadjustment pain. Am J Orthod Dentofacial Orthop, St. Louis, v.108, no. 6, p. 614-622, 1995

13. MA, T.; MARANGONI, R. D.; FLINT, W. In vitro comparison of debonding force and intrapulpal temperature changes during ceramic orthodontic bracket removal using a carbon dioxide laser. Am J Orthod Dentofacial Orthop, St. Louis, v. 111, no. 2 p. 203-210, 1997.

14. MELLO, J. B.; MELLO, G. P. S. Laser em Odontologia. 1. ed. São Paulo: Ed. Santos, 2001

15. MERCADANTE, M. M. N.: MARTINS, D. R.; VILLA, N.; SCAVONE, JÚNIOR, H.; COTRIM-FERREIRA, F. A. Comportamento da polpa dental humana frente à descolagem de braquetes cerâmicos com laser $\mathrm{CO}_{2}$ in vivo. R Dental Press Ortodon Ortop Facial, Maringá, v. 8, n.1, p.77-85, 2003.

16. NOEL, L.; REBELLATO, J.; SHEATS, R. D. The effect of argon laser irradiation on demineralization resistance of human enamel adjacent to orthodontic brackets: an in vitro study. Angle Orthod, Appleton, v. 73, no. 3, p. 249-258, 2003

17. RICKABAUGH, J. L.; MARANGONI, R. D.; McCAFFREY, K. K. Ceramic bracket debonding with the carbon dioxide laser. Am J Orthod Dentofacial Orthop, St. Louis, v. 110, no. 4 p. 388-393, 1996

18. ROBERTS-HARRY, D. Lasers in Orthodontics. Br J Orthod, London, v. 21, no. 3, p. 308-312, 1994

19. ROMEO, A.; CANAL, F.; ROMA, M.; DE LA HIGUERA, B.; USTRELL, J. M.; VON ARX, J. D. Holograms in orthodontics: a universal system for the production, development, and illumination of holograms for the storage and analysis of dental casts. Am J Orthod Dentofacial Orthop, St. Louis, v. 108, no. 4 P. 443-447, 1995.

20. SAITO, S.; SHIMIZU, N. Stimulatory effects of low-power laser irradiation on bone regeneration in midpalatal suture during expansion in the rat. Am J Orthod Dentofacial Orthop, St. Louis, v. 111 , no. 5, p. 525-532, 1997

21. STROBL, K.; BAHNS, T. L.; WILLHAM, L.; BISHARA, S. E STWALLEY, W. C. Laser-aided debonding of orthodontic ceramic brackets. Am J Orthod Dentofacial Orthop, St. Louis, v. 101, no. 2, p. 152-158, 1992

22. TALBOT, T. Q.; BLANKENAU, R. J.; ZOBITZ, M. E.; WEAVER, A. L.; LOHSE, C. M.; REBELLATO, J. Effect of argon laser irradiation on shear bond strength of orthodontic brackets: an in vitro study. Am J Orthod Dentofacial Orthop, St. Louis, v. 118 no. 3, p. 274-279, 2000.

\section{Endereço para correspondência}

Leniana Santos Neves

Alameda Dr. Octávio Pinheiro Brisolla n 9-75.

CEP: 17.012-901 - Bauru/SP

E-mail: leniananeves@uol.com.br 